\section{Immunoglobulin D multiple myeloma, plasma cell leukemia and chronic myelogenous leukemia in a single patient treated simultaneously with lenalidomide, bortezomib, dexamethasone and imatinib}

\author{
Naveed Ali, ${ }^{1}$ Peter V. Pickens, ${ }^{2}$ \\ Herbert E. Auerbach ${ }^{3}$ \\ Departments of ${ }^{1}$ Internal Medicine, \\ ${ }^{2}$ Hematology and Oncology, ${ }^{3}$ Pathology, \\ Abington Memorial Hospital/Abington- \\ Jefferson Health, PA, USA
}

\section{Abstract}

Multiple myeloma (MM) is a neoplastic lymphoproliferative disorder characterized by uncontrolled monoclonal plasma cell proliferation. Among different isotypes of MM, immunoglobulin D (IgD) MM is very rare, representing only 1 to $2 \%$ of all isotypes. Chronic myelogenous leukemia (CML) is a neoplastic myeloproliferative disorder of pluripotent hematopoietic stem cell, which is characterized by the uncontrolled proliferation of myeloid cells. An 88-year-old male was diagnosed simultaneously with IgD kappa MM and CML. A distinctive feature in this patient was the progression to plasma cell leukemia without any symptomatic myeloma stage. He was treated simultaneously with lenalidomide, bortezomib and imatinib. Synchronous occurrence of these rare hematological malignancies in a single patient is an exceedingly rare event. Multiple hypotheses to explain co-occurrence of CML and MM have been proposed; however, the exact etiological molecular pathophysiology remains elusive.

\section{Introduction}

Multiple myeloma (MM) has an annual incidence of 5.6 cases per 100,000 individuals in the US. ${ }^{1}$ Chronic myelogenous leukemia (CML) has an annual incidence of 1-2 cases per 100,000 individuals. ${ }^{2-4}$ Co-occurrence of these two disorders in one patient is an extremely rare event, described in a handful of cases in the literature. ${ }^{4-14}$ We describe an exceedingly rare case of simultaneous immunoglobulin D kappa MM with plasma cell leukemia (PCL) and CML which, to our knowledge, has not been reported in the literature.

\section{Case Report}

An 88-year-old male urologist with a history of atrial fibrillation, iron deficiency anemia and unprovoked pulmonary thromboembolism, presented to our hospital in January 2014 after a fall leading to rhabdomyolysis and quadriceps tendon rupture. Upon laboratory investigations, he was found to have normocytic normochromic anemia with a hemoglobin level of $9.8 \mathrm{~g} / \mathrm{dL}$, mean corpuscular volume of $94.3 \mathrm{fl}$ and reticulocyte count of $2.3 \%$. White blood cell count was $14.6 \times 10^{3}$ per $\mu \mathrm{L}$ (58\% neutrophils, $12 \%$ lymphocytes, $30 \%$ monocytes, $0 \%$ basophils and $0 \%$ eosinophils) and platelet count was $191 \times 10^{3}$ per $\mu \mathrm{L}$. Absolute monocyte count was elevated at $4.4 \times 10^{3}$ per $\mu \mathrm{L}$ (normal range $0.0-0.9 \times 10^{3}$ per $\mu \mathrm{L}$ ). Iron deficiency anemia and megaloblastic anemia due to hypovitaminosis B12 or folate were excluded. Presence of anemia and persistent monocytosis suggested underlying bone marrow pathology such as myelodysplasia or chronic myelomonocytic leukemia, and warranted a bone marrow examination.

A bone marrow aspirate and biopsy showed mild trilineage hypercellularity with myeloid to erythroid cell ratio of 4.7:1; however monocytes were not noted to be elevated (Figure 1A-C). Immunohistochemical stains demonstrated CD138 positive, CD20 positive, monoclonal kappa plasma cells accounting for $10 \%$ cellularity (Figure 1D,E). Flow cytometry demonstrated plasma cells positive for kappa chain, CD38, CD138, CD20, partial CD45, dim CD27 and CD200, and negative for CD19, CD56, CD117, CD28 and CD81. Cytogenetic analysis by FISH (fluorescence in situ hybridization) demonstrated translocation $\mathrm{t}(11 ; 14)$ (Figure $1 \mathrm{~F}$ ) and deletion chromosome 17p13 (TP53) but did not detect rearrangements of chromosome 1p or $1 \mathrm{q}$, gain of chromosome $5 \mathrm{q}$, deletion of chromosome 13 (or $13 q$ ), $t(14 ; 16)$ or $t(4 ; 14)$. Unexpectedly, karyotype analysis detected Philadelphia chromosome [t $(9 ; 22)(\mathrm{q} 34 ; \mathrm{q} 11)]$ in 8 out of 20 metaphases analyzed (Figure 1G). Serum protein electrophoresis and immunofixation showed immunoglobulin D kappa monoclonality with M-spike of $1 \mathrm{~g} / \mathrm{dL}$. A skeletal survey was unremarkable for any characteristic myeloma bony lesions, as was magnetic resonance imaging of the spine. Serum calcium and renal function were normal. Serum free kappa chains were elevated with an increased kappa:lambda chain ratio (Table 1). Immunoglobulin levels were depressed except for markedly elevated immunoglobulin D level (Table 1). At the same time, quantification of chimeric $B C R-A B L$ mRNA transcripts was done by reverse transcription polymerase chain reaction (RT-PCR). There were no plasma cells detected on peripheral smear examination. He was therefore diagnosed with $\operatorname{IgD}$
Correspondence: Naveed Ali, Abington Memorial Hospita//Abington-Jefferson Health, Department of Internal Medicine, 1200 Old York Road, Abington, 19001 PA, USA.

Tel.: +1.267.471.0165 - Fax: +1.215.481.4361.

E-mail: nali@abingtonhealth.org

Key words: Immunoglobulin D multiple myeloma; plasma cell leukemia; chronic myelogenous leukemia; bortezomib; imatinib.

Contributions: the authors contributed equally.

Conflict of interest: the authors declare no potential conflict of interest.

Received for publication: 4 November 2015. Revision received: 19 January 2016.

Accepted for publication: 19 January 2016.

This work is licensed under a Creative Commons Attribution-NonCommercial 4.0 International License (CC BY-NC 4.0).

(C) Copyright N. Ali et al., 2016

Licensee PAGEPress, Italy

Hematology Reports 2016; 8:6295

doi:10.4081/hr.2016.6295

kappa smoldering multiple myeloma (DurieSalmon stage I and International Staging System I) ${ }^{15}$ and chronic phase chronic myelogenous leukemia simultaneously. Therapy was not initiated and a watch and wait approach was implemented with serial clinical and laboratory assessments.

In May 2014, after two months of initial diagnosis, he developed profound fatigue, severe nocturnal sweating and low grade fevers. On laboratory evaluation, he had a sudden increase in white blood cell count to $40 \times 10^{3}$ per $\mu \mathrm{L}$, increase in serum kappa chain level and decrease in immunoglobulin levels (Table 1). A peripheral blood smear examination showed presence of circulating plasma cells, plasmacytoid lymphocytes and abnormal lymphocytes which were previously not present (Figure $1 \mathrm{H}$ ). He had rapidly progressed from an asymptomatic smoldering myeloma stage to PCL.

Therapy for MM and PCL was instituted as RVD (lenalidomide, bortezomib and dexamethasone) regimen that resulted in disappearance of plasma cells from peripheral circulation. Imatinib for treatment of CML was not started along with RVD to avoid potentially dangerous adverse effects from combined proteasome inhibitor and tyrosine kinase inhibitor therapy given patient's advanced age and co-morbidities. However, he exhibited worsening leukocytosis and thrombocytosis with counts as high as $90 \times 10^{3}$ per $\mu \mathrm{L}$ and $2069 \times 10^{3}$ per $\mu \mathrm{L}$, respectively. $B C R-A B L$ mRNA transcripts were found elevated at $180.38 \%$. He was subsequently started on imatinib (400 mg 
daily) for CML. Both RVD and imatinib were continued which he tolerated well with diarrhea as the only side effect which was well controlled with antidiarrheal medication.

Over the next few weeks, he demonstrated subjective improvement as well as improve- ment in free kappa chain, LDH, $B C R-A B L$ mRNA transcripts and immunoglobulin D levels. A trend of myeloma parameters longitudinally over time is summarized in Table 1. A complete clinical remission, however, was not achieved. The immunoglobulin levels contin- ued to decline. In September 2014, he was admitted to the hospital and succumbed to fulminant septic shock consequent to pneumonia.

Table 1. Laboratory myeloma parameters.

M-spike, g/dL

Free kappa chain level (normal range $3.3-19.4 \mathrm{mg} / \mathrm{L}$ )

Free lambda chain level (normal range $5.7-26.3 \mathrm{mg} / \mathrm{L}$ )

Free kappa/lambda ratio (normal range 0.26-1.65)

Beta 2 microglobulin (normal $<2.51 \mathrm{mg} / \mathrm{L}$ )

LDH (normal range 120-250 U/L)

Immunoglobulin G (normal range 694-1618 mg/dL)

Immunoglobulin A (normal range $81-463 \mathrm{mg} / \mathrm{dL}$ )

Immunoglobulin M (normal range $48-271 \mathrm{mg} / \mathrm{dL}$ )

Immunoglobulin D (normal < 179 mg/L)

MM, multiple myeloma; PCL, plasma cell leukemia; LDH, lactate dehydrogenase

\begin{tabular}{ccc}
1 & 1 & 0 \\
99.5 & 107.2 & 21.5 \\
6.4 & 8.1 & 10.8 \\
15.55 & 13.23 & 1.99 \\
\hline 2.56 & 3.53 & 4.61 \\
258 & 595 & 209 \\
433 & 312 & 267 \\
65 & 34 & 31 \\
49 & 38 & 32 \\
4640 & 3570 & 152
\end{tabular}

Table 2. Characteristics of cases of coexisting multiple myeloma and chronic myelogenous leukemia.

\begin{tabular}{|c|c|c|c|c|}
\hline Light chain & $\begin{array}{l}\text { Case } \\
\text { report }\end{array}$ & Age/ Sex & $\begin{array}{l}\text { Chronological } \\
\text { order }\end{array}$ & Treatment \\
\hline \multicolumn{5}{|r|}{ Immunoglobulin G } \\
\hline$\kappa$ & Pessach et al. ${ }^{5}$ & $63 / \mathrm{F}$ & $\mathrm{CML} \rightarrow \mathrm{MM}$ & CML: Imatinib, MM: bortezomib, lenalidomide, dexamethasone \\
\hline$\lambda$ & Pessach et al. ${ }^{5}$ & $68 / \mathrm{M}$ & $\mathrm{MM} \rightarrow \mathrm{CML}$ & MM: Vincristine, doxorubicin, dexamethasone, radiation, zoledronic acid, CML: imatinib \\
\hline$\kappa$ & Alsidawi et al. ${ }^{6}$ & $60 / \mathrm{M}$ & $\mathrm{MM} \rightarrow \mathrm{CML}$ & $\begin{array}{l}\text { MM: Radiation, lenalidomide, bortezomib, dexamethasone, CML/ AML - hydroxyurea, } \\
\text { dasatinib }\end{array}$ \\
\hline$\kappa$ & Ragupathi et al. ${ }^{7}$ & $62 / \mathrm{F}$ & $\mathrm{MM} \rightarrow \mathrm{CML}$ & $\begin{array}{l}\text { MM: Radiation, dexamethasone, bortezomib, cyclophosphamide, doxorubicin, } \\
\text { lenalidomide, CML: dasatinib }\end{array}$ \\
\hline$\kappa$ & Offiah et $a l^{8}$ & $71 / \mathrm{F}$ & $\mathrm{MM}+\mathrm{CML}$ & $\begin{array}{l}\text { CML: Imatinib, MM: melphalan, prednisolone, bortezomib, dexamethasone, lenalidomide, } \\
\text { cyclophosphamide }\end{array}$ \\
\hline$\kappa$ & Ide et $_{\text {al. }}{ }^{9}$ & $72 / \mathrm{F}$ & $\mathrm{CML} \rightarrow \mathrm{MM}$ & CML: Imatinib, MM: no treatment \\
\hline$\lambda$ & Garipidou et al. ${ }^{12}$ & $68 / \mathrm{M}$ & $\mathrm{CML} \rightarrow \mathrm{MM}$ & CML: INF-alpha, imatinib, MM: malphalan and prednisolone \\
\hline$\lambda$ & Wakayama et al. ${ }^{5}$ & $85 / \mathrm{F}$ & $\mathrm{MM}+\mathrm{CML}$ & \\
\hline$\kappa$ & Shwarzmeier et al. ${ }^{13}$ & 3 $66 / \mathrm{M}$ & $\mathrm{CML}+\mathrm{MM}$ & CML: Hydroxyurea, IFN-alpha, busulfan, MM: melphalan and prednisolone \\
\hline$\kappa$ & Nitta et al. ${ }^{5,7}$ & $70 / \mathrm{M}$ & $\mathrm{MM} \rightarrow \mathrm{CML}$ & CML: Hydroxycarbamide, MM: Not reported \\
\hline$\kappa$ & Tanaka et al. ${ }^{5,7}$ & $72 / \mathrm{F}$ & $\mathrm{CML}+\mathrm{MM}$ & CML: INF-alpha, hydroxycarbamide, MM: INF-alpha, Vindesine sulfate, prednisolone \\
\hline$\kappa$ & Klenn et al. ${ }^{5,7}$ & $71 / \mathrm{M}$ & $\mathrm{MM} \rightarrow \mathrm{CML}$ & MM: Radiation, malphalan, prednisone, CML: Hydroxyurea \\
\hline$\kappa$ & Boots et al. 5,7 & $58 / \mathrm{M}$ & $\mathrm{CML}+\mathrm{MM}$ & MM: Radiation, malphalan, prednisone, CML: hydroxyurea, busulfan, thioguanine \\
\hline$\kappa$ & Derghazarian et al. ${ }^{5,7}$ & 7 65/F & $\mathrm{CML} \rightarrow \mathrm{MM}$ & MM and CML: busulphan, Radiation, phenylalanine mustard \\
\hline \multicolumn{5}{|r|}{ Immunoglobulin A } \\
\hline$\lambda$ & Maerki et al. ${ }^{4}$ & $77 / \mathrm{M}$ & $\mathrm{CML}+\mathrm{MM}$ & MM: radiation, bortezomib, CML: anti CML treatment (not specified) \\
\hline$\kappa$ & Romanenko et al. ${ }^{5}$ & $64 / \mathrm{F}$ & $\mathrm{CML}+\mathrm{MM}$ & MM: bortezomib, dexamethasone, CML: Imatinib \\
\hline$\kappa$ & Michael et al. ${ }^{10}$ & $57 / \mathrm{F}$ & $\mathrm{CML} \rightarrow \mathrm{MM}$ & $\begin{array}{l}\text { CML: Imatinib, MM: thalidomide, dexamethasone, vincristine, liposomal doxorubicin, } \\
\text { bortezomib }\end{array}$ \\
\hline$\kappa$ & Galanopoulos et al. ${ }^{11}$ & $1176 / \mathrm{M}$ & $\mathrm{CML} \rightarrow \mathrm{MM}$ & CML: INF-alpha, imatinib, MM: malphalan and prednisolone \\
\hline$\kappa$ & Alvarez-Larran et al. ${ }^{1}$ & $1481 / \mathrm{M}$ & $\mathrm{CML}+\mathrm{MM}$ & MM: Malphalan and prednisone, CML: not reported \\
\hline \multicolumn{5}{|r|}{ Bence Jones protein } \\
\hline$\lambda$ & Yokota et al. $^{5}$ & $71 / \mathrm{M}$ & $\mathrm{CML} \rightarrow \mathrm{MM}$ & CML: Imatinib \\
\hline \multirow[t]{2}{*}{$\kappa$} & Nakagawa et al. $^{5}$ & $47 / \mathrm{M}$ & $\mathrm{MM} \rightarrow \mathrm{CML}$ & \\
\hline & MacSween et al. ${ }^{5}$ & $77 / \mathrm{M}$ & $\mathrm{MM} \rightarrow \mathrm{CML}$ & CML: 6-mercaptopurine, MM: None \\
\hline \multicolumn{5}{|r|}{ Immunoglobulin D } \\
\hline$\underline{\kappa}$ & Present Case & $88 / \mathrm{M}$ & $\mathrm{MM}+\mathrm{CML}$ & MM: RVD, CML: Imatinib \\
\hline
\end{tabular}

MM, multiple myeloma; CML, chronic myelogenous leukemia; INF-alpha, interferon alpha. 

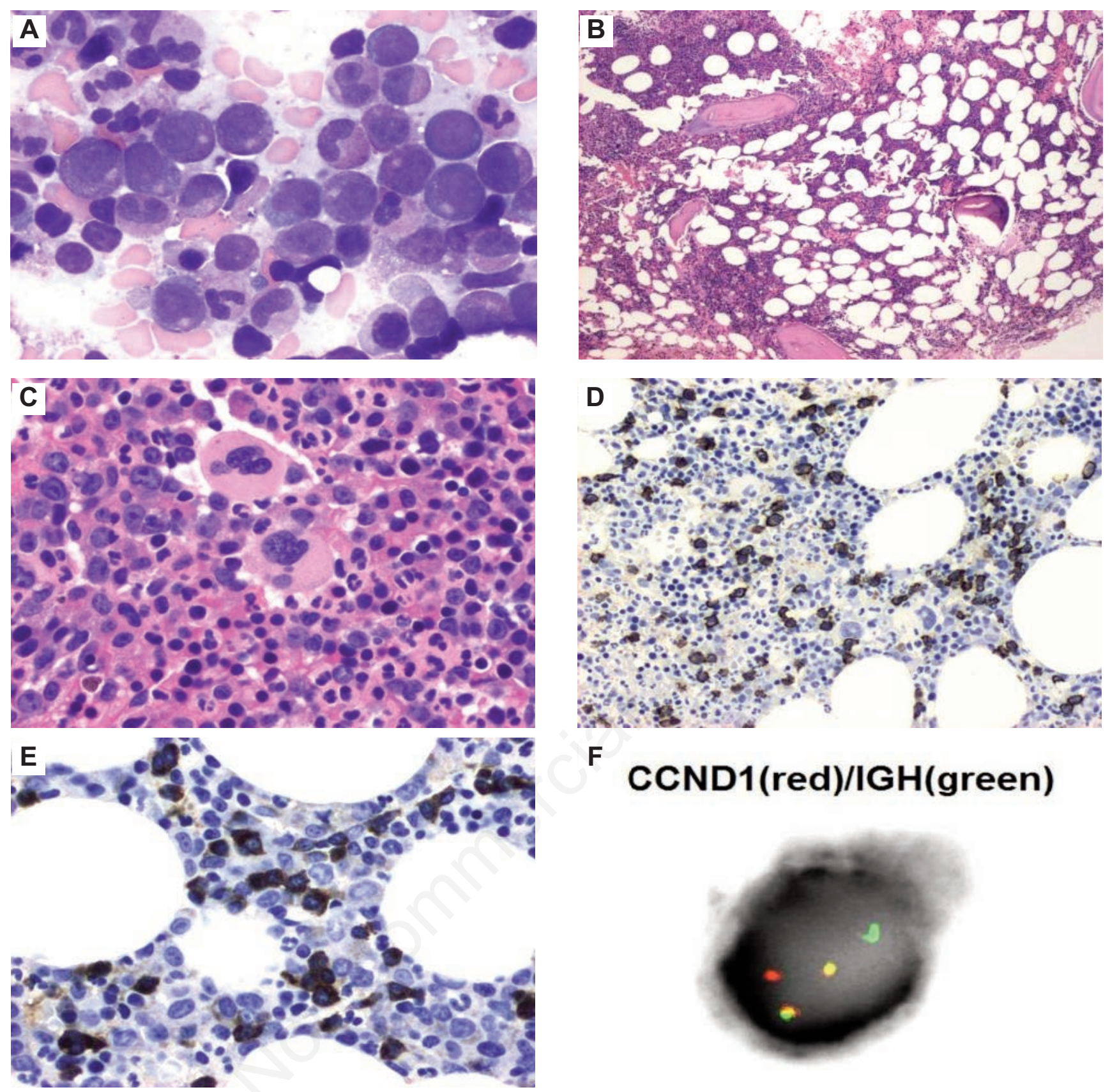

\section{$\mathbf{F}$ \\ CCND1(red)/IGH(green)}

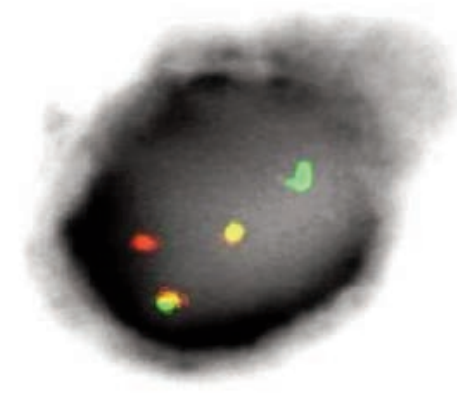

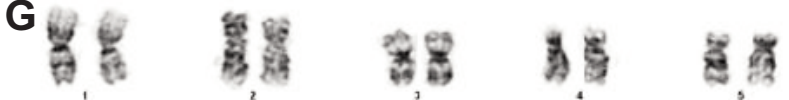

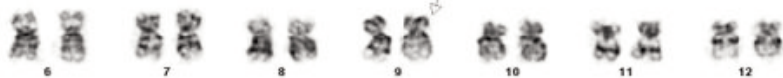

\begin{tabular}{|c|c|c|c|c|}
\hline$\Leftrightarrow \Leftrightarrow$ & $\Rightarrow 8$ & 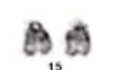 & $8 \%$ & 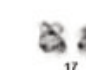 \\
\hline & 8 & & & \\
\hline
\end{tabular}

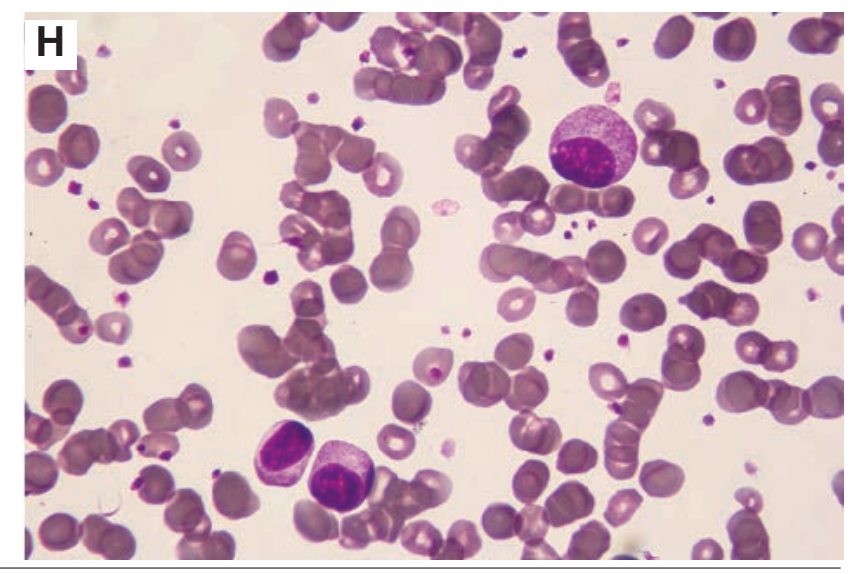

Figure 1. A) Myeloid and erythroid cells in bone marrow aspirate; B) bone marrow core biopsy showing mild hypercellularity; C) trilineage hematopoiesis; D) CD 138 positive plasma cells; E) kappa chain positive plasma cells; F) fluorescence in situ hybridization analysis showing 1 red signal (CCND1 or Cyclin D1), 1 green signal (IgH) and 2 yellow fusion signals $[\mathbf{t}(11 ; 14)]$; G) karyotype analysis showing translocation between chromosome 9 and 22 (arrows); H) peripheral smear showing plasma cells with eccentric nucleus and perinuclear halo. 


\section{Discussion}

Based on National Cancer Institute Surveillance, Epidemiology and End Results Program (SEER) data, there is an increased risk of secondary hematological malignancies after MM and CML. ${ }^{4,5,16,17}$ However, co-occurrence of these two disorders in one patient is exceedingly rare, described very infrequently in the literature (Table 2). ${ }^{4-14}$ Most of these cases were diagnosed sequentially with one disease preceding the other with varying time intervals between the two diagnoses. MM developed first in 7 cases, ${ }^{5-7}$ while CML was diagnosed first in 7 cases. ${ }^{5,9-12}$ Eight cases were diagnosed with MM and CML simultaneously. ${ }^{4,5,8,13,14}$ Our case is different from the prior-reported ones in several perspectives. First, to our knowledge, our patient is the only case of CML coexisting with immunoglobulin D MM. Among previously reported cases, 14 patients had immunoglobulin G myeloma, 5 patients had immunoglobulin A myeloma and 3 patients demonstrated light chain disease (Bence Jones protein) (Table 2). Although rare, IgD MM is prognostically unfavorable as the disease is usually advanced at diagnosis (Durie-Salmon stage III), has higher risk of extramedullary spread, poor prognosis and increased risk of transforming into plasma cell leukemia which is a rare and aggressive plasma cell disorder seen in only 1 to $4 \%$ of end stage myeloma patients. ${ }^{18-20}$ Secondly, M-spike was noted to be only $1 \mathrm{~g} / \mathrm{dL}$ in our patient. IgD myeloma tends to have low M-spike as compared to other myeloma types. ${ }^{18}$ In addition, IgD myeloma tends to express lambda light chains more commonly which is different in our case as the plasma cell clones were kappa chain restricted. ${ }^{18}$ Thirdly, the present patient received lenalidomide, bortezomib and imatinib simultaneously with minimal side effects. Only 3 cases were reported to have received proteasome inhibitors and tyrosine kinase inhibitors together with favorable adverse effect profile. ${ }^{5,6,8}$ Fourthly, our patient rapidly progressed to aggressive plasma cell leukemia. PCL superimposed on CML has been reported on very rare occasions. ${ }^{4,14}$ It is worth mentioning that our case progressed from smoldering MM to PLC without a transitional symptomatic myeloma stage.

Primary PCL (arising de novo) and secondary PCL (leukemic transformation of terminal stage relapsed or refractory MM) represent two distinct entities with different cytogenetic and molecular findings. ${ }^{19}$ Immunophenotype analysis showed CD20 positive plasma cells in our patient which is highly expressed in cases of PCL. Furthermore, CD27 was expressed which confers plasma cells an antiapoptotic capability, another observation in PCL. ${ }^{20}$ Our patient lacked expressions of CD117 and
CD56. Lack of CD56, a cell adhesion molecule, is a characteristic finding in PCL that causes egression of plasma cells from bone marrow microenvironment to the peripheral circulation. ${ }^{19,20}$ The presence of CD20/CD27 and absence of CD117/CD56 are more common in primary PCL than in MM (or secondary PCL). IgH gene rearrangements, in particular translocation $\mathrm{t}(11 ; 14)$, are also more common in primary PCL than secondary PCL. ${ }^{19,20}$ Based on lack of intermediary symptomatic myeloma stage, presence of $\mathrm{t}(11 ; 14)$ and immunophenotypic observations described above, we may hypothesize that our patient developed primary PCL rather than secondary PCL.

Investigators have proposed several potential pathophysiologies to explain the synchronous or metachronous presence of MM and CML. Firstly, a common malignant progenitor stem cell capable of differentiating into both myeloid and lymphoplasmacytic cell lineages could potentially transform into either MM or CML, which can explain the present case. Schwarzmeier et al. studied this hypothesis in a patient with simultaneous MM and CML. In vitro analysis of the isolated myeloma cells did not demonstrate $B C R-A B L$ fusion gene, thereby concluding that MM and CML did not arise from a common malignant clone. ${ }^{13}$

Tanaka et al., on the other hand, reported a case where $97 \%$ of the marrow nucleated cells exhibited $B C R-A B L$ fusion gene. Plasma cells constituted $19 \%$ of the bone marrow cellularity suggesting that some of these cells harbored $B C R-A B L$ fusion gene. ${ }^{4}$

Secondly, tumorigenesis after exposure to environmental carcinogens such as ionizing radiation and chlorinated solvents is a wellknown phenomenon. ${ }^{17}$ A common insult to the bone marrow can cause irreparable DNA damage potentially leading to neoplasms of myeloid and lymphoid origin. We postulate that out patient, being a urologist, may have had exposure to ionizing radiation during his professional life that manifested in the form of simultaneous occurrence of MM and CML.

Thirdly, treatment of first malignancy with cytotoxic drugs or radiation can potentially lead to secondary malignant transformation. ${ }^{4,17}$ In this regard, the role of melphalan and cylophosphamide in developing secondary malignancies is well documented. Various secondary malignancies in lenalidomide-treated patients have also been described. ${ }^{17}$ Nevertheless, this hypothesis cannot be extrapolated to our patient because he did not receive any of such prior treatments.

Fourthly, epigenetic upregulation or downregulation of molecular pathways as a result of progression or treatment of multiple myeloma could potentially pave ways for malignant myeloid clones to emerge. These malignant cells acquire antiapoptotic ability and mechanisms to escape immune surveillance in the background of immunoparesis rendered by multiple myeloma. In addition, chronic antigenic stimulation due to multiple etiological factors may be involved in the development of simultaneous myeloid and lymphoid neoplasms. ${ }^{17}$ Other factors such as genotypic poylmorphism may also be involved.

\section{Conclusions}

In conclusion, the association between MM (or plasma cell leukemia) and CML may be multifactorial. Survival has improved in patients with MM and CML, due to the advent of novel therapies. With improving survival, increased incidence of secondary malignancies is emerging. Therefore, secondary malignancies in patients with MM and CML should be vigilantly investigated if there is a high index of suspicion. Based on our and others experiences, ${ }^{4-6}$ we can conclude that simultaneous treatment with proteasome inhibitors and tyrosine kinase inhibitors may be compatible. In any case, further investigation on this aspect is required and encouraged.

\section{References}

1. Palumbo A, Anderson K. Multiple myeloma. N Engl J Med 2011;364:1046-60.

2. Sawyers CL. Chronic myeloid leukemia. N Engl J Med 1999;340:1330-40.

3. Goldman JM, Melo JV. Chronic myeloid leukemia. Advances in biology and new approaches to treatment. N Engl J Med 2003;349:1451-64.

4. Maerki J, Katava G, Siegel D, et al. Unusual case of simultaneous presentation of plasma cell myeloma, chronic myelogenous leukemia, and a jak2 positive myeloproliferative disorder. Case Rep Hematol 2014;2014:738428.

5. Pessach I, Bartzis V, Tzenou T, et al. Multiple myeloma and chronic myelogenous leukemia; an uncommon coexistence in 2 patients, with literature review. Ann Hematol Oncol 2015;2:1030.

6. Alsidawi S, Ghose A, Qualtieri J, Radhakrishnan N. A case of multiple myeloma with metachronous chronic myeloid leukemia treated successfully with bortezomib, dexamethasone, and dasatinib. Case Rep Oncol Med 2014;2014:962526.

7. Ragupathi L, Najfeld V, Chari A, et al. A case report of chronic myelogenous leukemia in a patient with multiple myeloma and a review of the literature. Clin Lymphoma Myeloma Leuk 2013;13:175-9.

8. Offiah C, Quinn JP, Thornton P, Murphy 
PT. Co-existing chronic myeloid leukaemia and multiple myeloma: rapid response to lenalidomide during imatinib treatment. Int J Hematol 2012; 95:451-2.

9. Ide M, Kuwahara N, Matsuishi E, et al. Uncommon case of chronic myeloid leukemia with multiple myeloma. Int $\mathrm{J}$ Hematol 2010;91:699-704.

10. Michael M, Antoniades M, Lemesiou E, et al. Development of multiple myeloma in a patient with chronic myeloid leukemia while on treatment with imatinib mesylate for 65 months. Oncologist 2009;14:1198200.

11. Galanopoulos A, Papadhimitriou SI, Kritikou-Griva E, et al. Multiple myeloma developing after imatinib mesylate therapy for chronic myeloid leukemia. Ann Hematol 2009;88:281-2.

12. Garipidou V, Vakalopoulou S, Tziomalos K. Development of multiple myeloma in a patient with chronic myeloid leukemia after treatment with imatinib mesylate. Oncologist 2005;10:457-8.

13. Schwarzmeier JD, Shehata M, Ackermann $\mathrm{J}$, et al. Simultaneous occurrence of chronic myeloid leukemia and multiple myeloma: evaluation by FISH analysis and in vitro expansion of bone marrow cells. Leukemia 2003;17:1426-8.

14. Alvarez-Larrán A, Rozman M, Cervantes F. Simultaneous occurrence of multiple myeloma and chronic myeloid leukemia. Haematologica 2001;86:894.

15. Rajkumar SV, Dimopoulos MA, Palumbo A, et al. International Myeloma Working Group updated criteria for the diagnosis of multiple myeloma. Lancet Oncol 2014;15:e538-48

16. Curtis RE, Freedman DM, Ron E, et al. New malignancies among cancer survivors: SEER Cancer Registries, 1973-2000.
National Cancer Institute. NIH Publ. No. 05-5302. Bethesda, MD, 2006.

17. Thomas A, Mailankody S, Korde N, et al. Second malignancies after multiple myeloma: from 1960s to 2010s. Blood 2012;119: 2731-7.

18. Pandey S, Kyle RA. Unusual myelomas: a review of IgD and IgE variants. Oncology (Williston Park) 2013;27:798-803.

19. Van de Donk NWCJ, Lokhorst HM, Anderson KC, Richardson PG. How I treat plasma cell leukemia. Blood 2012;120: 2376-89.

20. De Larrea CF, Kyle RA, Durie BG, et al. Plasma cell leukemia: consensus statement on diagnostic requirements, response criteria, and treatment recommendations by the International Myeloma Working Group (IMWG). Leukemia 2013;27:780-91. 